\title{
Proceeding
}

Supplementary Issue: Winter Conferences of Sports Science. International Conference of Engineering, Innovation Technology and Applied Science.

\section{Relationship between dysfunctional attitudes and mental health in psychiatric nurses}

\author{
RAANA JAFARIZADEH ${ }^{1}$, BAHMAN AGHAIE ${ }^{2}$, MEHRDAD AZARBARZIN³ ${ }^{3}$ NARGES SADEGHI ${ }^{4}$ \\ ${ }^{1}$ Department of Medicine, Ardabil Branch, Islamic Azad University, Ardabil, Iran \\ ${ }^{2}$ Nursing Department, Faculty of Nursing and Midwifery, Qom University of Medical Sciences, Qom, Iran \\ ${ }^{3}$ Nursing and Midwifery Sciences Development Research Center, Najafabad Branch, Islamic Azad University, \\ Najafabad, Iran \\ ${ }^{4}$ Community Health Research Center, Islamic Azad University, Isfahan, Iran
}

\begin{abstract}
Introduction: Psychiatric nurses face numerous acute stresses in their workplace that can adversely affect their job performance and the quality of their health services. Many factors affect the mental health of psychiatric nurses, including dysfunctional attitudes. hence, the present study aims to investigate the relationship between dysfunctional attitudes and the mental health in psychiatric nurses. Methodology: A descriptive study was conducted on 96 psychiatric nurses working in a psychiatric hospital in Isfahan in 2018. The participant were selected using convenience sampling and the data were collected using the Dysfunctional Attitudes Scale and the Symptom Checklist-25. Findings: The results indicated an inverse relationship between the mean scores of mental health and dysfunctional attitudes $(p<.001)$. The mean score of dysfunctional attitudes was a significant predictor of the mean score of mental health $(p<.05)$. Mental health and dysfunctional attitudes of psychiatric nurses did not exhibit a significant relationship with their age and work experience $(p>.05)$. In addition, there was no significant difference between males and females in terms of the mean scores of mental health and dysfunctional attitudes $(p>.05)$. Conclusion: The study findings confirm the role of dysfunctional attitudes in mental health in psychiatric nurses. The health system authorities are recommended to identify and control the causes of dysfunctional attitudes among psychiatric nurses in order to reduce the risks and consequences and improve the quality of nursing services. Training courses for nurses on the importance and necessity of mental health, as well as the risks and consequences of dysfunctional attitudes, can be effective in this regard.
\end{abstract}

Keywords: Dysfunctional attitude; Mental health; Psychiatric nurses.

\section{Cite this article as:}

Jafarizadeh, R., Aghaie, B., Azarbarzin, M., \& Sadeghi, N. (2020). Relationship between dysfunctional attitudes and mental health in psychiatric nurses. Journal of Human Sport and Exercise, 15(2proc), S437-S443. doi:https://doi.org/10.14198/ihse.2020.15.Proc2.34

Corresponding author. Community Health Research Center, Islamic Azad University, Isfahan, Iran.

E-mail: Bahman.aghai@gmail.com

Supplementary Issue: Winter Conferences of Sports Science. International Conference of Engineering, Innovation Technology and Applied Science.

JOURNAL OF HUMAN SPORT \& EXERCISE ISSN 1988-5202

(C) Faculty of Education. University of Alicante

doi:10.14198/jhse.2020.15.Proc2.34 


\section{INTRODUCTION}

Mental health is one of the components of health assessment in different societies. It is also considered one of the basics of health by the World Health Organization (WHO) (World Health Organization, 2014). In fact, it is a dynamic state of inner balance that enables individuals to harmoniously use their abilities. Flexibility, the ability to cope with adverse life events, and the body-mind coordination are some components of mental health, each partially contributing to the state of inner balance (Galderisi et al., 2015). Mental health is one of the components of a healthy lifestyle, with numerous factors contributing to its proper or improper formation (Kaplan \& Sadock, 2015). One of the factors affecting the health of employees is their job status. Although almost all professions are associated with work-related stresses, nurses usually face more stressful situations (Villani et al., 2013). They are the main service providers in the health care system whose performance can greatly affect public health (Tavakkoli et al., 2015). Studies have shown that nursing is an emotional and physical occupation requiring a high risk of experiencing stress, anxiety, and depression. Hence, it is very important to maintain the physical and mental health of the nursing staff in their workplace (Perry et al., 2015). Psychiatric nursing is the appointed position of a nurse that specializes in mental health and cares for people of all ages experiencing mental illnesses or distress (Ong et al., 2017). Psychiatric nurses pay special attention to caring for the mental and physical health of patients, and their role has expanded over the years. If the mental and physical health of nurses is not protected, most of them may experience mental health disorders, which can negatively affect the health care provided by them. It can be stated that occupational stressors in psychiatric nurses are potentially different from those in other people (Yada et al., 2015). The performance of psychiatric nurses in psychiatry departments can be complex and challenging. The patients admitted to these departments usually suffer from the most severe mental illnesses and require complicated needs and care. They may be convicted and referred by the court. On the other hand, patient violence against other patients and the medical staff, suicidal attempts and unstable behaviours of patients, unpredictable nature of mental disorders, and working in an environment full of different demands and challenges impose great pressures on nurses and have unpleasant consequences such as stress, job stress, job burnout and job dissatisfaction (Fereidooni Moghadam, 2013). Yavari et al. showed that the mental health of psychiatric nurses was significantly lower than that of nurses working in physical care units (Yavari et al., 2014). Psychiatric nursing has always been considered a less attractive job option due to several reasons such as negative attitudes towards people with mental illnesses, job-related negative experiences, and no communication with people suffering from mental disorders (Ong et al., 2017).

The role of mental attitudes and processes in many behavioural disorders and problems has been demonstrated. It has been also shown that irrational beliefs can exacerbate one's suffering. Based on past experiences, dysfunctional attitudes make one prone to psychological disorders. Such attitudes are actually one's biased assumptions and beliefs about oneself, the surrounding world, and the future. According to Beck, people use inflexible criteria and perfectionism to judge themselves and others. Since these criteria are inflexible, extreme, and change-resistant, they are considered insufficient (A'zami et al., 2015). In other words, one's cognitive appraisal, schemas, and attitudes towards a position affect one's level of adaptation (Wise \& Barnes, 2009). Studies have shown that one's attitude towards oneself, the surrounding environment, and others greatly affects the way one copes with stresses (Mostafaee et al., 2012). Stresses themselves may cause no anxiety but the interaction between multiple stresses and how one perceives and responds to the environment can endanger one's mental health (Valizade, 2006). Such attitudes generally increase one's vulnerability to mental disorders and prepare one to interpret highly negative and dysfunctional situations (Dehbaneh \& Bahrainian, 2018). The effects of dysfunctional attitudes on stress, anxiety, and depression have been investigated but few studies have dealt with its effects on the mental health of psychiatric nurses. 
Identification of the factors affecting mental health is one of the most important issues in organizational promotion. Considering the significance of promoting the mental health of psychiatric nurses and the effects of dysfunctional attitudes on the quality of nursing services, the present study aims to investigate the relationship between dysfunctional attitudes and the mental health of psychiatric nurses to be a basis for the development of mental health promotion for psychiatric nurses.

\section{METHODOLOGY}

This was a descriptive study conducted in Isfahan, Iran in 2018. The study population consisted of all nurses working in a psychiatric hospital. The inclusion criteria were no history of taking psychiatric drugs, working in the psychiatry department, at least one year of work experience as a nurse, and willingness to participate in the study. Based on these criteria, 96 nurses from the morning, evening, and night shifts of the studied hospital were selected as the sample by using convenience sampling. The data were collected using the Dysfunctional Attitudes Scale (DAS-26) and the Symptom Checklist-25 (SCL-25). Developed by Weissman and Beck in 1978, DAS-26 consists of 4 subscales as follows: perfectionism, seeking others' attention (confirmation), seeking others' satisfaction, and vulnerability and functional evaluation. The items are scored based on a 7-point Likert scale (from totally agree to totally disagree). The minimum and maximum scores obtainable on this scale are 26 (low dysfunctional attitude) and 182 (high dysfunctional attitude), respectively (Ebrahimi et al., 2007). SCL-25 is a single-component checklist with 25 items that are scored based on a 5point Likert scale. In this study, mental health refers to the participants' scores on SCL-25. The minimum and maximum scores on this checklist are 25 and 125, respectively, and higher scores indicate the better status of mental health (Najarian \& Davoodi, 2001). The Data were statistically analysed in SPSS-18 by using the Pearson correlation coefficient and the independent t-test at the .05 level of significance.

\section{FINDINGS}

The mean age of participants was $35.44 \pm 7.55$ and their mean work expertise was equal to $9.36 \pm 6.58$. In terms of gender, 25 participants $(26.6 \%)$ were male and $69(73.4 \%)$ were female. The results showed that the total score of dysfunctional attitudes was equal to $88.10 \pm 21.04$. The level of dysfunctional attitudes was low, moderate, and high in 3 participants (3.2\%), 90 participants (95.7\%), and only one participant (1.1\%), respectively. In addition, the mean mental health score of participants was obtained $99.26 \pm 17.76$. The results demonstrated that the level of mental health was moderate and high in 22 participants (23.4\%) and 72 participants (76.6\%). The Pearson correlation coefficient showed that there was an inverse relationship between the mean scores of mental health and dysfunctional attitudes $(p<.001)$. In other words, the mean mental health score increased with a decrease in the total score of dysfunctional attitudes (Table 1).

Table 1. Pearson correlation coefficient between the mean scores of mental health and dysfunctional attitudes.

\begin{tabular}{|l|c|c|}
\hline \multirow{2}{*}{ Variable } & \multicolumn{2}{|c|}{ Mental health score } \\
\cline { 2 - 3 } & $\mathrm{R}$ & $\mathrm{P}$ \\
\hline Dysfunctional attitudes score & -0.677 & $<.001$ \\
\hline
\end{tabular}

Linear regression analysis showed that the total score of dysfunctional attitudes was a significant predictor of mental health $(p<.05)$ (Table 2). 
Table 2. Linear regression analysis to predict mental health score by total score of dysfunctional attitudes.

\begin{tabular}{|l|c|c|c|c|}
\hline Variable & Raw coefficients & Standardized coefficients & T-value & $\mathbf{p}$-value \\
\hline Dysfunctional attitudes score & 0.472 & -0.559 & 6.75 & $<.001$ \\
\hline
\end{tabular}

Pearson correlation coefficient indicated that mental health and dysfunctional attitudes of psychiatric nurses did not exhibit a significant relationship with their age and work experience $(p>.05)$. Based on the independent t-test, there was no significant difference between males and females in terms of the mean scores of mental health and dysfunctional attitudes $(p>.05)$.

\section{DISCUSSION}

Considering the importance of mental health in psychiatric nurses, the present study aimed to investigate the relationship between dysfunctional attitudes and the mental health of psychiatric nurses. The findings revealed a significant inverse relationship between dysfunctional attitudes and the mental health of psychiatric nurses. In other words, the mean mental health score increased with a decrease in the total score of dysfunctional attitudes. The results also showed that dysfunctional attitudes were a significant predictor of mental health. Consistent with the present study, the findings of previous studies have reported a significant negative relationship between dysfunctional attitudes and mental health. Moallemi et al. found a significant negative correlation between mental health and dysfunctional attitudes (Moallemi et al., 2010). The findings of Hajializadeh et al. indicated a significant and positive correlation between dysfunctional attitudes and depression in addicts (Bahreinian et al., 2008). Musa al-Rezaei et al. reported that dysfunctional attitudes were significantly and positively correlated with stress, anxiety, and depression. Mehdiyar et al. showed that dysfunctional attitude was a significant predictor of depression (Mehdiyar et al., 2019). The findings of Calloway, Cheng and Yusooff , and Stolow were consistent with those of the present study (Calloway, 2010; Cheng \& Yusooff, 2010; Stolow, 2011).

Increased anxiety and depression are among the factors that can negatively affect the mental health of individuals. Zul-Rahim et al. reported that there was a significant negative relationship between dysfunctional attitudes and components of psychological well-being among university students (Zul-Rahim \& Beyrami, 2015). Declined psychological well-being can affect mental health and cause disastrous consequences. Farmani et al. stated that dysfunctional attitudes and irrational beliefs can cause emotional turmoil. People who have pessimistic views on life issues always find themselves surrounded by unpleasant events and evaluate their personal events and experiences as stressful. These attitudes make individuals prone to different diseases (Farmani et al., 2014). Mazloom et al. argue that pessimistic attitudes are one of the causes of psychological disorders in psychiatric nurses, and the interaction between different stresses and the way psychiatric nurses perceive ad react to the surrounding environment can endanger their mental health (Mazloom et al., 2012).

These results suggest that dysfunctional attitudes are activated by stressors and affect one's mental health. Given the importance and role of the mental health of psychiatric nurses in patient safety and health, this study sought to investigate the factors affecting mental health and pave the way for the mental health promotion by preventing traumatic factors towards a deep understanding of mental health and the relevant factors. Dysfunctional attitudes are one of the factors associated with mental health. Therefore, it is necessary to identify and control it. The study findings also suggested that the mental health and dysfunctional attitudes of psychiatric nurses did not exhibit a significant relationship with their age and work experience. In addition, there was no significant difference between males and females in terms of the mean scores of mental health and dysfunctional attitudes. 


\section{CONCLUSION}

The study results can be helpful in understanding the factors affecting the mental health of nurses working in psychiatry wards. The health system authorities are recommended to identify and control the factors causing dysfunctional attitudes among psychiatric nurses in order to reduce the risks and consequences and improve the quality of nursing services. It is recommended that psychiatric nurses be trained to prevent and manage stresses. Increased dysfunctional attitudes can negatively affect and impair the mental health of nurses. Therefore, mental health specialists are recommended to pay special attention to dysfunctional attitudes and coping strategies and plan to prevent and reduce complications and risks of dysfunctional attitudes. In addition, training courses for nurses on the importance and necessity of mental health and its components, as well as the risks and consequences of dysfunctional attitudes, can be effective in this regard.

\section{ETHICAL CONSIDERATIONS}

The present study was approved by the Ethics Committee if the Islamic Azad University of Isfahan (Khorasgan) (IR.IAU.KHUISF.REC.1397.164). The participants were briefed on the study objectives and procedures and assured that their information would be kept confidential. Additionally, the participants could leave the study whenever they desired.

\section{ACKNOWLEDGMENTS}

The present paper was extracted from a thesis approved by the Islamic Azad University of Isfahan (Khorasgan). The authors would like to thank the authorities and nurses of the psychiatric hospital who helped us in the present research project.

\section{REFERENCES}

A'zami, Y., Doostian, Y., Mo'tamedi, A., Massah, O., \& Heydari, N. (2015). Dysfunctional attitudes and coping strategies in substance dependent and healthy individuals. Iranian Rehabilitation Journal, 13(1), 55-51.

Bahreinian, S. A., Hajializadeh, K., Moddares, M., \& Naziri, G. (2008). Comparison of Dysfunctional Attitudes in Addicts and Normal People and its Psychological Consequences. Journal of Addiction Research.

Calloway, C. S. (2010). Cognitive Vulnerabil Symptoms in Young Adolescents, ProQuest Dissertations \& Theses (PQDT).

Cheng, S. K., \& Yusooff, F. (2010). Investigating the Moderating and Mediating Effects of Dysfunctional Attitudes and Self-Esteem on the Relationship between Social Support and Depression among Late Adolescents in Klang Valley Malaysia: A Moderated Mediation Model. International Journal of Education and Information Technologies, 2(4), p. 73-82.

Dehbaneh, M. A., \& Bahrainian, S. A. (2018). The comparison between early maladaptive schemas and dysfunctional attitudes and coping strategies in people with Body Dysmorphic Disorder and healthy people in a study population in Tehran. World Family Medicine Journal: Incorporating the Middle East Journal of Family Medicine, 99(5641), 1-11. https://doi.org/10.5742/mewfm.2018.93208

Ebrahimi, M., Neshatdoust, H. T., Kalantari, M., Molavi, H., \& Assadollahi, H. (2007). Contribution of Dysfunctional Attitudes and General Health to Predicting the Odds of Depression. Journal of Shahrekord University of Medical Sciences, 9(4), 52-58. 
Farmani, S., Tajodini, A., Ghaednia, A., \& Masoumi, S. S. (2014). Attachment styles, Coping Styles for Stressful Situations, and Suicidal Ideation based on Dysfunctional Attitudes of Students of Kharazmi University: A Comparative Study. Journal of Counseling and Psychotherapy, 3(11).

Fereidooni Moghadam, M. (2013). Designing and validiation of nursing model of patients with severe mental illness in psyehiatric ward [Dissertation]. Ahvaz: Ahvaz Jundishapur University of Medical Sciences.

Galderisi, S., Heinz, A., Kastrup, M., Beezhold, J., \& Sartorius, N. (2015). Toward a new definition of mental health. World Psychiatry, 14(2), 231-233. https://doi.org/10.1002/wps.20231

Kaplan, B. J., \& Sadock, V. A. (2015). Synopsis of Psychiatry Bahavioural Science, Updated with DSM5.

Ong, H. L., Seow, E., Chua, B. Y., Xie, H., Wang, J., Lau, Y. W., ... \& Subramaniam, M. (2017). Why is psychiatric nursing not the preferred option for nursing students: A cross-sectional study examining pre-nursing and nursing school factors. Nurse education today, 52, 95-102. https://doi.org/10.1016/j.nedt.2017.02.014

Mazloom, N., Pak, S., Dadgari, A., Safari, M., \& Nadi, M. (2012). Evaluation of Dysfunctional Attitudes, Coping Strategies, and Resilience in Hypertensive Patients and Healthy Individuals; The Fourth International Congress on Psychosomatic Medicine.

Mehdiyar, M., Taghavi, S. M., \& Goudarzi, M. A. (2019). Prediction of Depression Based on Material Affiliations, Attachment to God, and Dysfunctional Attitudes; Journal of Psychology and Religion, No. 45.

Moallemi, S, Bakhshaei, N. G., \& Raghibi, M. (2010). Relationship of Mental Health and Spiritual Intelligence with Dysfunctional Attitudes among Students of University of Sistan and Baluchestan. Journal of Mental Health Principles, 12(4), 9-702.

Mostafaee, F. A. T. E. M. E. H., Roshan, R., \& Jodeiri, B. (2012). Comparing dysfunctional attitude and coping response between patients with peptic ulcer disease and healthy control. The Journal of Qazvin University of Medical Sciences, 16(1), 44-50.

Najarian, B., \& Davoodi, A. (2001). Development and Validation of SCL-25 (Short form of SCL-90-R). Journal of Psychology, 5(2).

Perry, L., Lamont, S., Brunero, S., Gallagher, R., \& Duffield, C. (2015). The mental health of nurses in acute teaching hospital settings: a cross-sectional survey. BMC nursing, 14(1), 15. https://doi.org/10.1186/s12912-015-0068-8

Stolow, D. (2011). A prospective examination of Beck's cognitive theory of depression in university students in mainland China (Doctoral dissertation, Rutgers University-Graduate School-New Brunswick).

Tavakkoli, S., Asaadi, M. M., Pakpour, A. H., \& Hajiaghababaei, M. (2015). Environmental psychology effects on mental health job satisfaction and personal well being of nurses. Iranian journal of psychiatry, 10(3), 158.

Valizade, Z. (2006). Compare of mental health and performance in university. M.Sc. Dissertation. Tehran: Tehran University, College of psychology, 23-50. (Persian).

Villani, D., Grassi, A., Cognetta, C., Toniolo, D., Cipresso, P., \& Riva, G. (2013). Self-help stress management training through mobile phones: An experience with oncology nurses. Psychological Services, 10(3), 315. https://doi.org/10.1037/a0026459

Wise, E., \& Barnes R. (2009). The relationship among life events, dysfunctional attitudes, and depression. Cognit Ther Res, 10, 257-66.

World Health Organization. (2014). Mental and Neurological Disorders. Available from: https://www.who.int/mental_health/en/ 
Yada, H., Lu, X., Omori, H., Abe, H., Matsuo, H., Ishida, Y., \& Katoh, T. (2015). Exploratory study of factors influencing job-related stress in Japanese psychiatric nurses. Nursing research and practice. https://doi.org/10.1155/2015/805162

Yavari, M., Shamsaei, F., \& Yazdanbakhsh, K. (2014). Comparison psychiatrics nurses' burnout and general health with critical care (ICU) nurses. Quarterly Journal of Nursing Management, 3(1),12. (Full Text in Persian).

Zul-Rahim, R., \& Beyrami, M. (2015). Relationship between Dysfunctional Attitudes and Psychological Well-being among Students of the Islamic Azad University of Tabriz. Journal of Community Health, 9 (3). 